\title{
Reliability Improvements to Centrifugal Pump Performance in Conjunction with Inducers, CFD Comparative Study
}

\author{
Said A. F. Hawash ${ }^{1}$, Dalia M. S. El Gazzar ${ }^{1}$ and Mohamed A. El Samanoudy ${ }^{2}$ \\ 1. Mechanical \& Electrical Research Institute, National Water Research Center, Qallubiya, Al-Kanater13621, Egypt \\ 2. Faculty of Engineering, Ain Shams University, Cairo 11566, Egypt
}

\begin{abstract}
In this research, centrifugal pump unit was analysed to study the effects of using inducers on its performance. Hydraulic tests were done to obtain the optimum hydraulic performance before and after using inducer. Two types of inducers were used in this work (axial, helical). For this purpose, a test rig was specially designed with a pump system to investigate the parameters under consideration. Plots (H-Q) curves, (B.P.-Q) curves and ( $\eta-Q)$ curves were used to show the effect of pump performance with and without inducers. The present study introduces a simulation of centrifugal pump performance in conjunction with inducers using CFD (Computational Fluid Dynamics), to compare it with experimentally observed values. The model investigates the impact of using inducers on the head and flow of the pump. The results of the CFD model and experimental are correlated well. Furthermore, the results help the decision makers of the pumps for future developments in pump performance assuring safe and reliable running condition of the water pumps. Also it may be used for more applications of larger head and flow pumps.
\end{abstract}

Key words: Centrifugal pump, axial \& helical inducers, hydraulic performance, CFD simulation, NPSH.

\section{Introduction}

Inducer is an axial impeller which is installed in the same shaft with the impeller of the pump, in front of pump. The main goal of inducer is generating an additionally specific energy on the inlet of the impeller of the pump. The inducer is used usually for the pumps with sever suction condition. It will be replaced after a certain operating time and that is why the technology for its execution has to be simple and economical. In case that using an inducer with a pumping head $H$, the level of the minimum pressure from the impeller of the pump is rising from correspondence with this pumping head [1]. The reliability of pumps was improved when the original stainless steel impeller and inducer were replaced by

Corresponding author: Said A. F. Hawash, researcher and head of Control and Cathodic Protection Dep., research fields: hydraulics of groundwater wells, aquifers, water wells design, irrigation gates, barrages control and maintenance, pump selection, operations, control and performances. E-mail: saidhawash64@yahoo.com. titanium, which is to raise the output shaft critical speed above the pump operating speed. These improvements have lowered the shaft vibration and greatly improved the reliability of the pump. Synchronous vibration continues to be a problem [2]. The cavitation phenomena start causing both of main pump head and/or efficiency to be reduced significantly, then generates vibration and noise. In order to avoid these phenomena, the inlet of the pump is fitted with a special rotor called an inducer, which can operate satisfactorily with extensive cavitation [3].

Rotational energy is imparted to the fluid as it is propagated up the inducer blades to the impeller inlet. Once the fluid has enough rotational energy it beings to rotate with the inducer blade and no longer moves up towards the impeller inlet. Using grooves or vanes on the inducer housing in a counter rotational direction to the inducer, will catch the rotating fluid. The fluid will be pushed up in the grooves or along the vanes towards the impeller inlet. This improved 
NPSHR (Net Positive Suction Head Required) by approximately $1 \mathrm{~m}$ of head at the rated flow. The design of inducers is critical to achieving high suction performance in industrial pumps and rocket engine turbo pumps. However, the conventional design approach, based on blade angles, often causes unstable pump operation. Instabilities such as strong inlet backflow and rotating cavitation in inducers may cause mechanical failures in pumps and the entire pumping system [4].

\section{Experimental Test Procedures}

Hydraulic tests were done to evaluate pump unit performance with and without conditions of using inducers. Firstly, the whole pump performance was analyzed before using inducer. Then, two types of inducers (helical-axial) were installed to study their effect on the hydraulic performance of the pump unit. The inducer was mounted on the threaded area of the rotor assembly (taking the place of the impeller nut) and operates as a low (NPSH) axial flow impeller in series with the main pump impeller [5]. Flow rate, suction and delivery pressure heads, power consumption, and overall efficiency are measured and calculated to determine hydraulic performance at the different operating conditions. BEP (best efficiency points) are determined by different operating speed for the pumping unit providing optimum hydraulic performance.

The test rig as shown in Fig. 1 is a closed circuit where the pump sucks from and delivers water to a big tank $\left(4 \mathrm{~m}^{3}\right)$. Two manometers with an error reading of $( \pm) 1 \%$ are mounted on the suction and delivery pipes to measure the pump head, while the calibrated flow-meter, with an error reading of $( \pm) 2 \%$ is mounted on the delivery pipe to measure the pump discharge, the discharge is controlled by means of a sluice valve.

The designed specifications of the pump are, 45 $\mathrm{L} / \mathrm{sec}$. discharge, $27 \mathrm{~m}$ head, $30 \mathrm{~kW}$ motor power, and 1,500 rpm motor speed as shown in Fig. 2.

\section{Hydraulic Results and Analysis}

A complete set of readings were taken at different discharges from shut-off to full open delivery valve, to cover the entire range of operation of the pump with and without inducers. Flow rate, suction and delivery pressure heads, power consumption, and overall efficiency are measured and calculated to determine hydraulic performance at the different conditions. Plots (Q-H) curves, (Q-P) curves and (Q- $\eta$ ) curves are used to show the effect of pump performance with and without inducers and for different parameters of helical and axial inducers. Hydraulic measurements were taken before using inducer as shown in Table 1 .

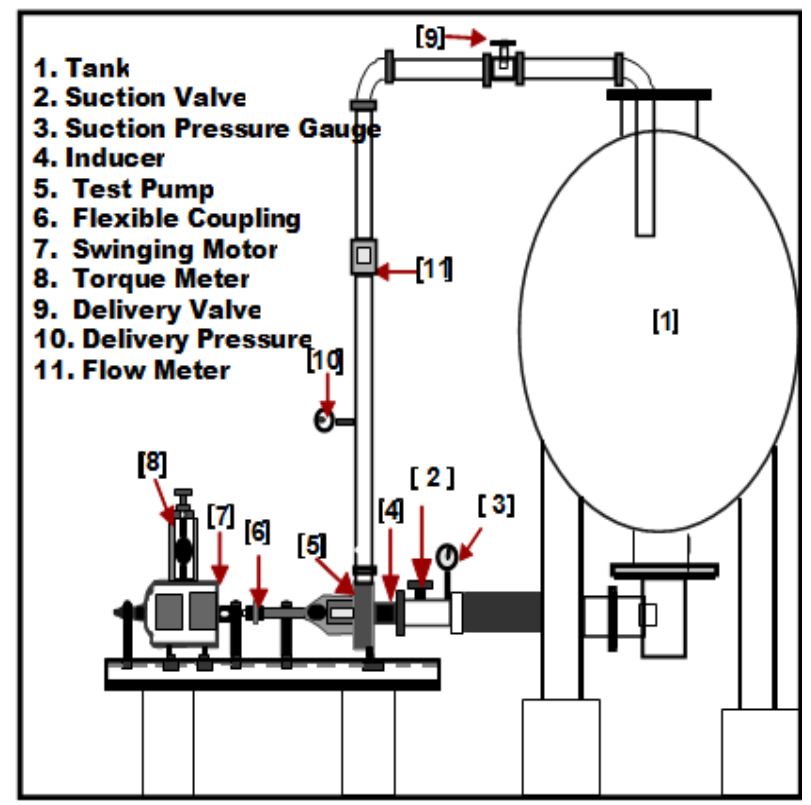

Fig. 1 Experimental test rig.

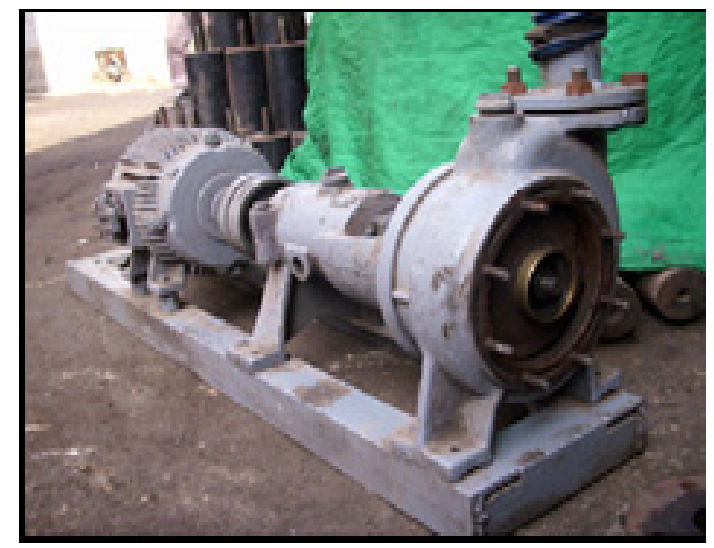

Fig. 2 Centrifugal pump used in the experimental test. 
Table 1 Hydraulic measurement results before using inducer.

\begin{tabular}{lllll}
\hline No. & $\begin{array}{l}\text { Flow } \\
(\mathbf{L} / \mathbf{s})\end{array}$ & $\begin{array}{l}\text { Total head } \\
(\mathbf{m})\end{array}$ & $\begin{array}{l}\text { Electric power } \\
(\mathbf{K w})\end{array}$ & $\begin{array}{l}\text { Overall eff. } \\
\mathbf{( \% )}\end{array}$ \\
\hline 1 & 65.4 & 12.72 & 26.2 & 33.55 \\
2 & 61.5 & 16.93 & 25.5 & 42.58 \\
3 & 58.8 & 19.04 & 24.5 & 44.79 \\
4 & 54 & 23.6 & 23.6 & 52.95 \\
5 & 50.2 & 24.66 & 23 & 53.07 \\
6 & 40.1 & 29.92 & 21.7 & 54.21 \\
7 & 29.1 & 33.79 & 19.1 & 50.47 \\
8 & 15.3 & 36.59 & 15.2 & 36.11 \\
\hline
\end{tabular}

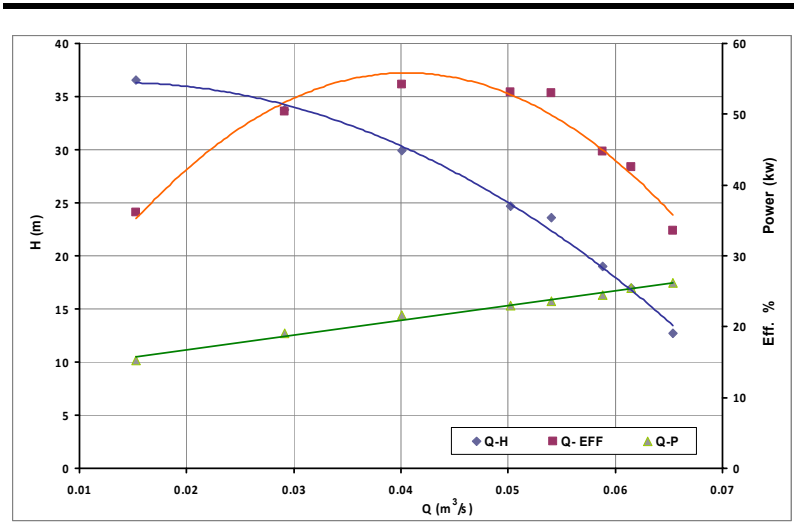

Fig. 3 Pump performance curve without inducer.

Performance curve for H-Q relation, static efficiency and consumed power, is shown in Fig. 3.

After that, inducer was mounted on the threaded area of the rotor assembly (taking the place of the impeller nut) and operates as a low (NPSH) axial flow impeller in series with the main pump impeller. Two types of inducers (axial \& helical) were installed as shown in Fig. 4. The axial inducer was manufactured with 4 blades with inlet and outlet blade angles $(\beta 1-\beta 2)$ of $\left(11.5^{\circ}-29.5^{\circ}\right)$ [5]. The helical inducer was manufactured with 4 turns and angle of $12^{\circ}$ and then, hydraulic measurements were repeated as shown in inducer Fig. 5.

\subsection{Firstly}

Axial inducer was installed and hydraulic measurements were taken as shown in Table 2 . Complete performance curve for H-Q relation, static efficiency and consumed power, is shown in Fig. 6. It could be seen that the head increased about $18 \%$. The maximum overall efficiency was improved and increased to be $68 \%$.

\subsection{Secondly}

The helical inducer was manufactured with 4 turns and angle of $12^{\circ}$, as shown in Fig. 7.

The helical inducer was installed and hydraulic measurements were taken as shown in Table 3. The

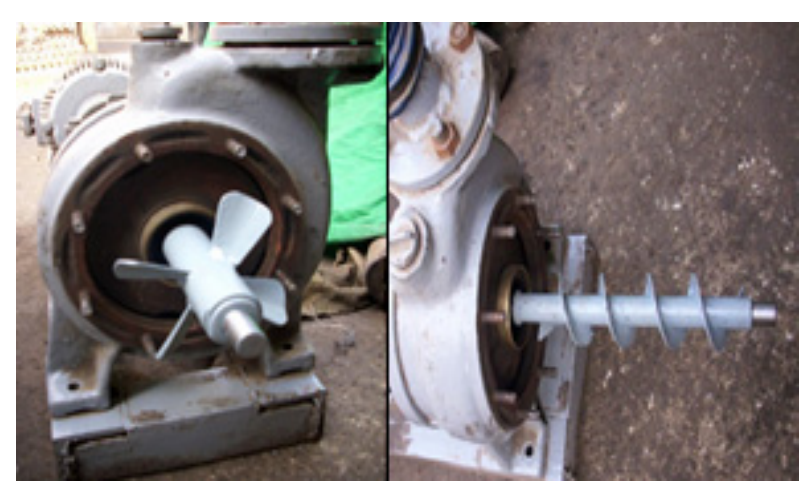

Fig. 4 Axial and helical inducers in conjunction with tested pump, respectively.

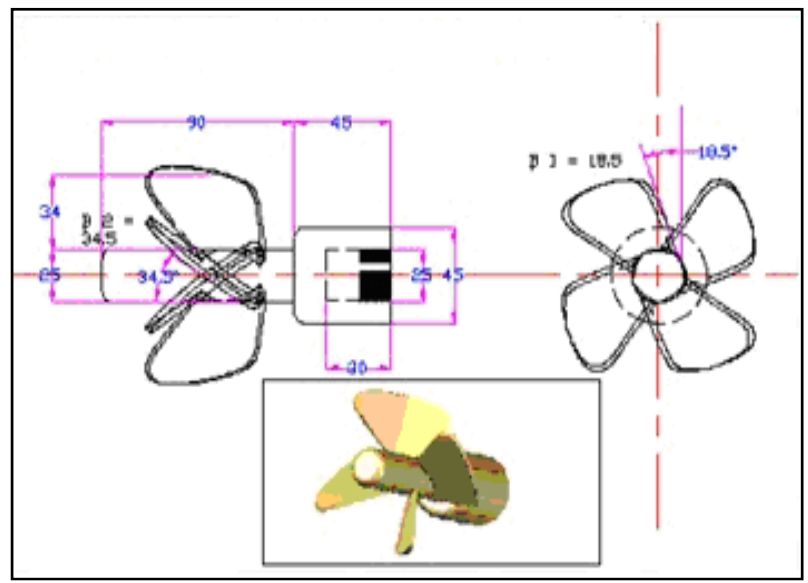

Fig. 5 Design parameters of an axial inducer.

Table 2 Hydraulic measurement results using axial inducer.

\begin{tabular}{lllll}
\hline No. & $\begin{array}{l}\text { Flow rate } \\
\left(\mathbf{m}^{\mathbf{3}} / \mathbf{s}\right)\end{array}$ & $\begin{array}{l}\text { Head } \\
(\mathbf{m})\end{array}$ & $\begin{array}{l}\text { Electric power } \\
(\mathbf{K w})\end{array}$ & $\begin{array}{l}\text { Overall eff. } \\
(\mathbf{\%})\end{array}$ \\
\hline 1 & 0.0654 & 17.7 & 26.8 & 38.72 \\
2 & 0.0615 & 20.33 & 26.5 & 48.84 \\
3 & 0.0588 & 24.60 & 26.3 & 59.47 \\
4 & 0.054 & 27.87 & 25.8 & 64.62 \\
5 & 0.0502 & 31.98 & 25.3 & 66.82 \\
6 & 0.0401 & 36.81 & 23.1 & 68.00 \\
7 & 0.0291 & 40.74 & 20.5 & 65.27 \\
8 & 0.0153 & 44.63 & 15.8 & 41.12 \\
\hline
\end{tabular}



Inducers, CFD Comparative Study

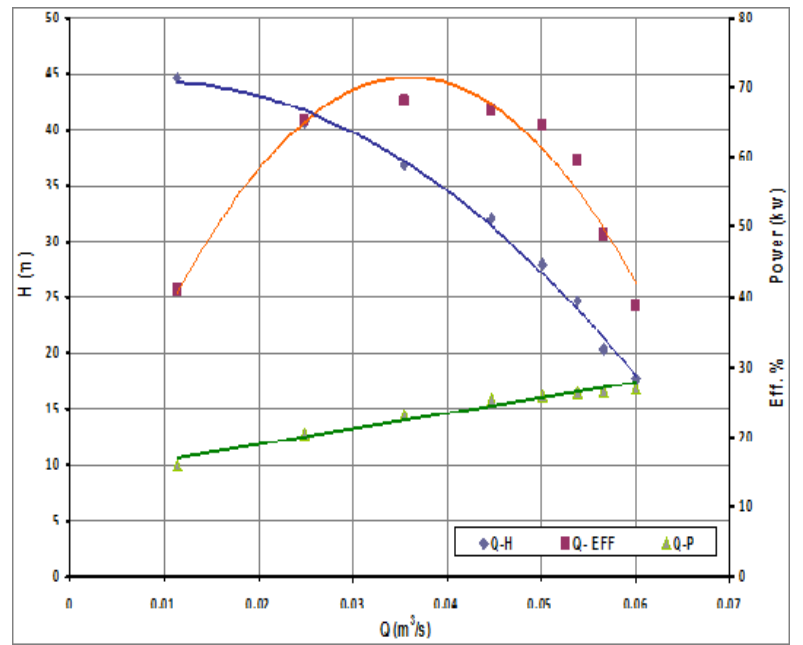

Fig. 6 Pump performance curve using axial inducer.

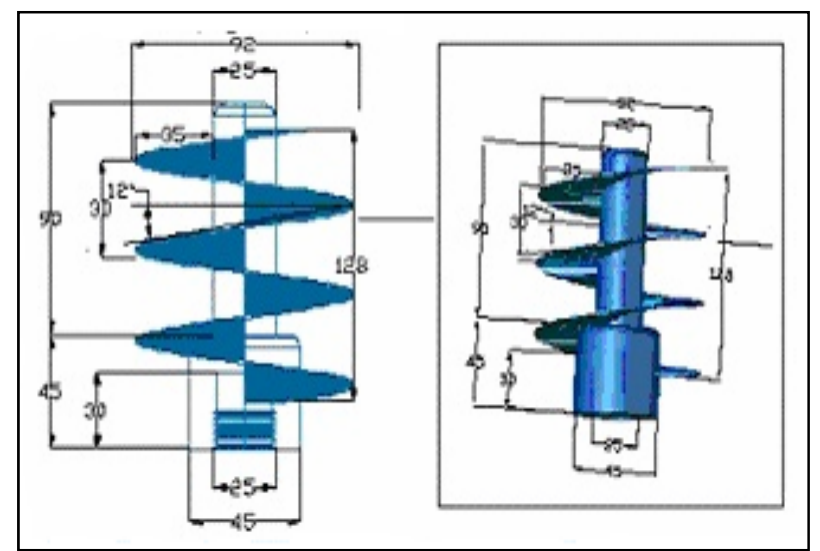

Fig. 7 Design parameters of helical inducer.

Table 3 Hydraulic measurement results using helical inducer.

\begin{tabular}{lllll}
\hline No & $\begin{array}{l}\text { Flow rate } \\
\left(\mathbf{m}^{\mathbf{3}} / \mathbf{s}\right)\end{array}$ & $\begin{array}{l}\text { Head } \\
(\mathbf{m})\end{array}$ & $\begin{array}{l}\text { Electric power } \\
(\mathbf{K w})\end{array}$ & $\begin{array}{l}\text { Overall } \\
\text { eff. }(\mathbf{\%})\end{array}$ \\
\hline 1 & 0.0654 & 16.37 & 26.8 & 38.2 \\
2 & 0.0615 & 22.03 & 26.5 & 57.61 \\
3 & 0.0588 & 26.14 & 26.3 & 58.89 \\
4 & 0.054 & 29 & 25.8 & 63.91 \\
5 & 0.0502 & 33.51 & 25.3 & 70.63 \\
6 & 0.0401 & 38.35 & 23.1 & 71.59 \\
7 & 0.0291 & 42.84 & 20.5 & 64.95 \\
8 & 0.0153 & 45.75 & 15.8 & 42.84 \\
\hline
\end{tabular}

results of hydraulic measurements using helical inducer are shown in Fig. 8. So, using helical inducer improved overall efficiency to reach $71.59 \%$ and increased head about $20 \%$.

Experimental work illustrated that using helical inducer enhanced the increasing of pump efficiency

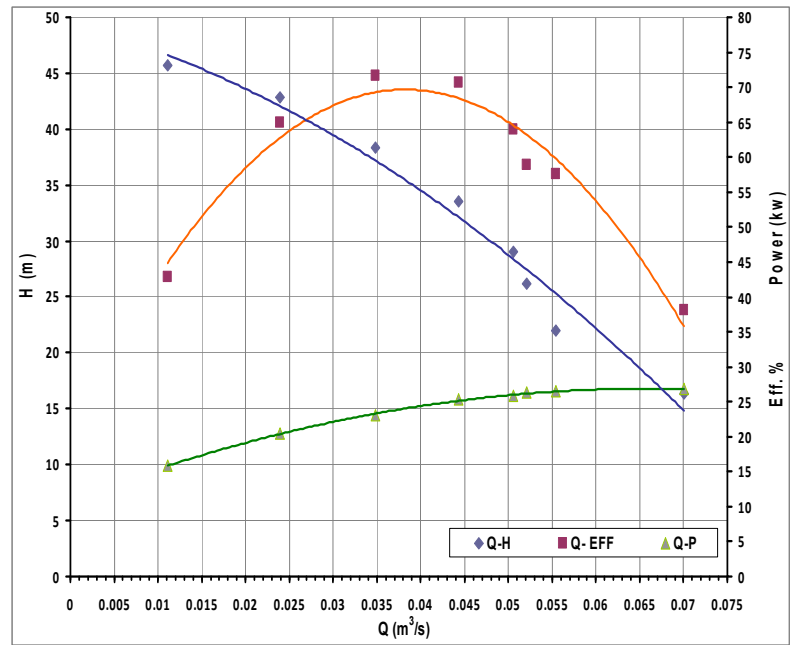

Fig. 8 Pump performance curve using helical inducer.

about $26 \%$, increased head about $20 \%$, whereas using axial inducer improved the increasing of pump efficiency reach $22 \%$ and increased head about $18 \%$. A CFD model will be done to compare experimental results with its numerical ones and show degree of correlation with the experimental values [6].

\section{Mathematical Modeling}

The CFD (computational fluid dynamic) model of the pump in conjunction with inducer is a crucial part of this study. The model investigates the impact of using inducer on the head and flow curves. The results of this phase have a huge impact on the quality of the results obtained by experimental setup and the mathematical model developed. The main problem was to model the geometry of the inducer in conjunction with the pump. Actually the CFD part of this study is only to find out the correlation between the experimentally observed data and data generated by the CFD model. If the CFD model thus develops to establish good correlation with the observed data, it could be used to analyze the various types of the system without fabricating the physical model. From a purely numerical perspective the geometric complexity of the problem is that it demands the full power of modern CFD to solve the equations of motion and turbulence models in domains that involve multiple surfaces [7]. 
Table 4 Change in head and efficiency values due to using helical and axial inducers.

\begin{tabular}{llllll}
\hline & $\begin{array}{l}\text { Without } \\
\text { inducer }\end{array}$ & $\begin{array}{l}\text { Axial } \\
\text { inducer }\end{array}$ & $\begin{array}{l}\text { Change } \\
\%\end{array}$ & $\begin{array}{l}\text { Helical } \\
\text { inducer }\end{array}$ & $\begin{array}{l}\text { Change } \\
\%\end{array}$ \\
\hline $\begin{array}{l}\text { Effici. } \\
\text { (\%) }\end{array}$ & 53.07 & 68 & $\underline{21.96}$ & 71.59 & $\underline{25.87}$ \\
$\begin{array}{l}\text { Head } \\
\text { (m) }\end{array}$ & 36.59 & 44.63 & $\underline{18.01}$ & 45.75 & $\underline{20.02}$ \\
\hline
\end{tabular}

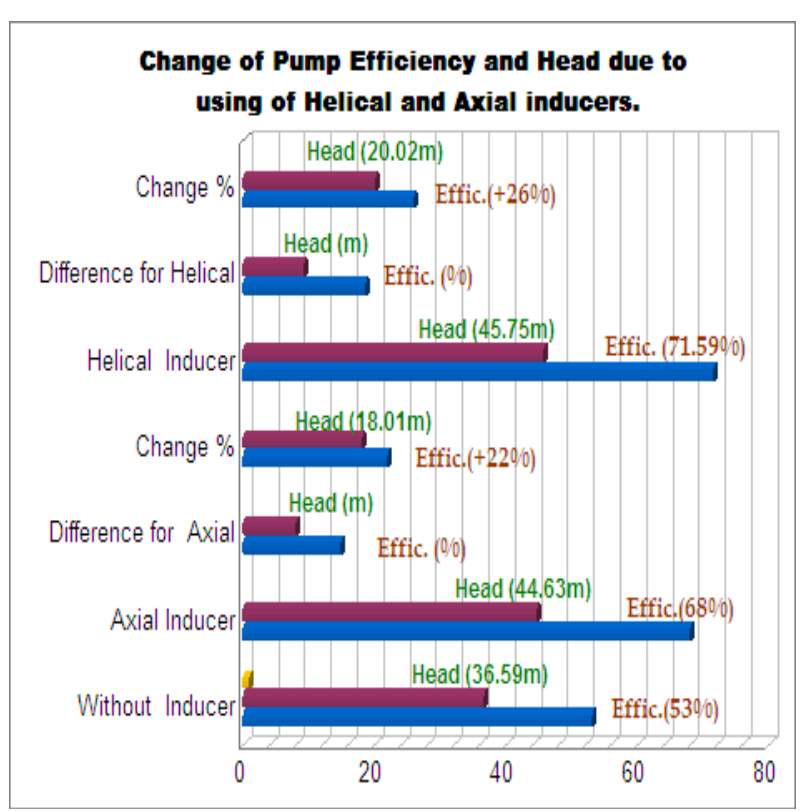

Fig. 9 Increasing head and efficiency values by using helical and axial inducers.

\subsection{Numerical Simulation and Building Model}

The numerical simulation has been made after the completion of experimental measurements. The simulation has been made using CFD code i.e. FLUENT software under ANSYS version 12.0, commercial code with solving 3D flow. The FLUENT model serves as a tool that discretizes and solves governing equations for specifics geometrics using a set of finite volume [8].

\subsection{Geometry Design}

The basic three-dimensional geometry is prepared using CFturbo. The generated grid using GAMBIT. The meshes required for the calculations were generated and checked by using the CFD preprocessing package, GAMBIT. Due to the geometry of the pump is very complex, unstructured tetrahedral mesh is used. "EquiAngle Skew" and
"EquiSize Skew" of the grid were all less than 0.95, so the grid quality is good. Fig. 10 shows the 3D model and wall grid of the calculation region with 7 blades.

\subsection{Numerical Simulation for Pump Model}

FLUENT was used to simulate the inner flow field under non cavitations' condition. The standard k- $\varepsilon$ turbulence model and SIMPLEC algorithm are applied to solve the RANS equations. The simulation is steady and moving reference frame is applied to take into account the impeller-volute interaction. Convergence precision of residuals is $10-5$.

\subsection{Governing Equations}

The incompressible flow through the rotating impeller is solved in a moving frame of reference with constant rotational speed equal the rotational speed of the impeller. The flow through the stationary parts of the pump is solved in an inertial reference frame. The governing equations for the impeller are formulated below:

$$
\begin{gathered}
\nabla \rho u_{r}=0 \\
\nabla \rho u_{r}+2 \rho \Omega * u_{r}+\rho \Omega * \Omega * r= \\
-\nabla P+\mu_{e f f} \nabla^{2} u_{r}
\end{gathered}
$$

Where $\rho$, is the density of the fluid, $P$ is the static pressure, $u_{r}$ is the vector fluid velocity in the rotating system, $\Omega$ is the rotational speed and $\mu_{\text {eff }}$ is the dynamic effective viscosity which is a linear combination of laminar and turbulent viscosity derived from $k-\varepsilon$ model of turbulence. The last two

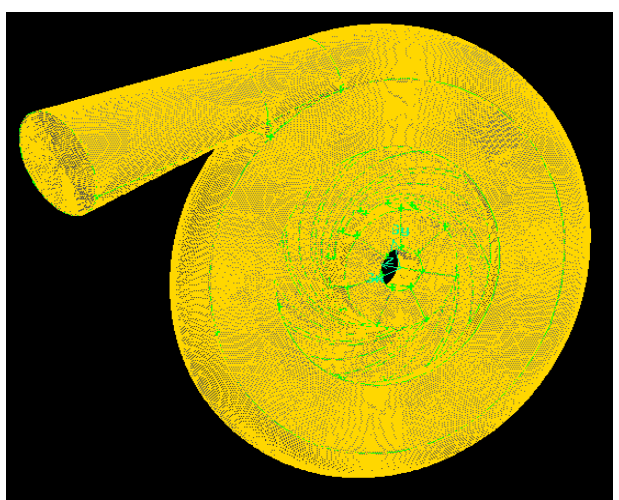

Fig. 10 The pump 3D model and wall grid. 
terms in the left hand side of Eq. (2) are the effects of the Coriolis and centrifugal forces due to the rotating frame of reference. For the stationary parts of the centrifugal pump, the governing equations are formulated in the stationary reference frame. The continuity equation remains the same, but the momentum equation reduces to:

$$
\nabla \rho u=-\nabla P+\mu_{e f f} \nabla^{2} u
$$

Where, $u$ is the vector fluid velocity in the stationary frame of reference. The turbulence of the flow is modeled with standard $k-\varepsilon$ model that is rated as the most used model that combines simplicity, robustness and reasonable accuracy. Moreover, it has been tested in a wide range of industrial flows showing satisfactory results. The differential transport equations for the turbulence kinetic energy and turbulence dissipation rate are:

$$
\begin{gathered}
\nabla \rho u k=\nabla\left[\left[\mu+\frac{\mu_{t}}{\sigma_{k}}\right] \nabla k\right]+G_{k}-\rho \varepsilon \\
\nabla \rho u \varepsilon=\nabla\left[\left[\mu+\frac{\mu_{t}}{\sigma_{t}}\right] \nabla \varepsilon\right]+C_{1 k} \frac{\varepsilon}{k} G_{k}-C_{2 \varepsilon} \rho \frac{\varepsilon^{2}}{k} \\
\mu_{t}=\rho C_{m} \frac{k^{2}}{\varepsilon}
\end{gathered}
$$

Where, $u$ is the local velocity vector, $k$ is the turbulent kinetic energy, $\varepsilon$ is the dissipation rate, $\mu$ is the laminar viscosity, $\mu_{t}$ is the turbulent viscosity, $G_{k}$ represents the generation of turbulent kinetic energy due to the mean velocity gradients, $\sigma k$ and $\sigma \varepsilon$ are the turbulent. Prandtl numbers and $C_{1 k}=1.44$, $C_{2 \varepsilon}=1.92$ and $C_{m}=0.09$ are the constants of the model.

\subsection{Boundary Conditions}

In the present study, mass flow rate and outflow boundary conditions were used for the inlet and outlet, respectively. Outer walls were stationary but the inner walls were rotational. There were interfaces between the stationary and rotational regions. Non slip boundary conditions have been imposed over the impeller blades and walls, the volute casing and the inlet wall and the roughness of all walls are considered $100 \mu \mathrm{m}$. The turbulence intensity at the inlet totally depends on the upstream history of flow. Since the fluid in the suction tank is undisturbed, the turbulence intensity for all conditions is considered $5 \%$. Water was used as a working fluid in ambient condition.

\section{Results and Discussion}

\subsection{Main Results}

Case 1 (pump without inducer): The hydraulic measurement results before using inducer can be illustrated as follows in Table 5 and Figs. 11 and 12. These values show that the static pressure increases gradually from inlet to outlet, the pressure difference between the outlet and the inlet is different. Heads upstream of the impeller can be computed by the pressure difference.

Case 2 (pump in conjunction with axial inducer): The hydraulic measurement results using axial inducer can be illustrated as follows in Table 6 and Figs. 13

\begin{tabular}{|c|c|c|c|}
\hline No. & Case & Velocity (m/s) & Pressure (psi) \\
\hline 1 & For impeller (inlet) & 4 & 1.2 \\
\hline 2 & $\begin{array}{l}\text { For impeller (outlet) } \\
\text { or (volute inlet ) }\end{array}$ & 16 & 26 \\
\hline 3 & For volute (outlet) & 6 & 34 \\
\hline
\end{tabular}
and 14.

Table 5 Hydraulic measurement results before using inducer.

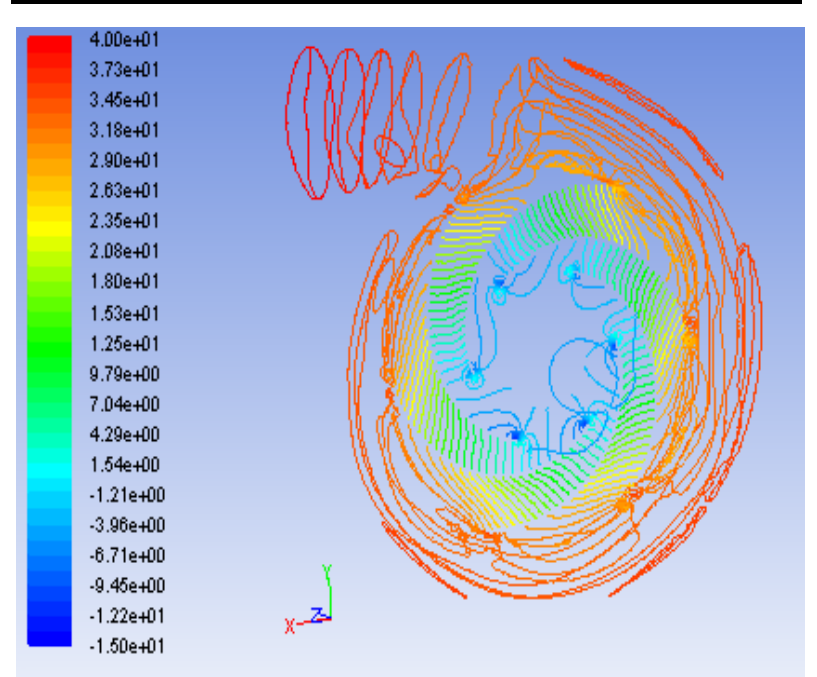

Fig. 11 Static pressure contour of case 1. 

Inducers, CFD Comparative Study

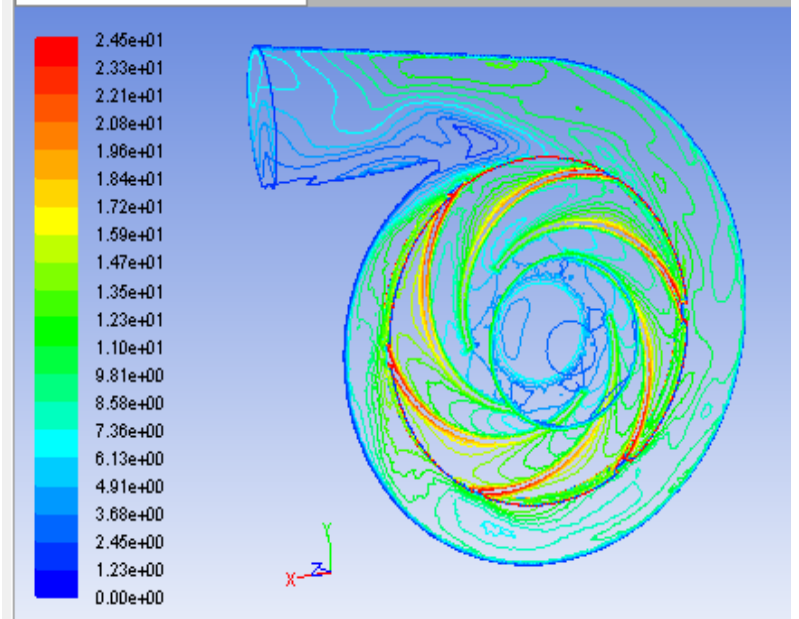

Fig. 12 Velocity contour of case 1.

Table 6 Hydraulic measurement results using axial inducer.

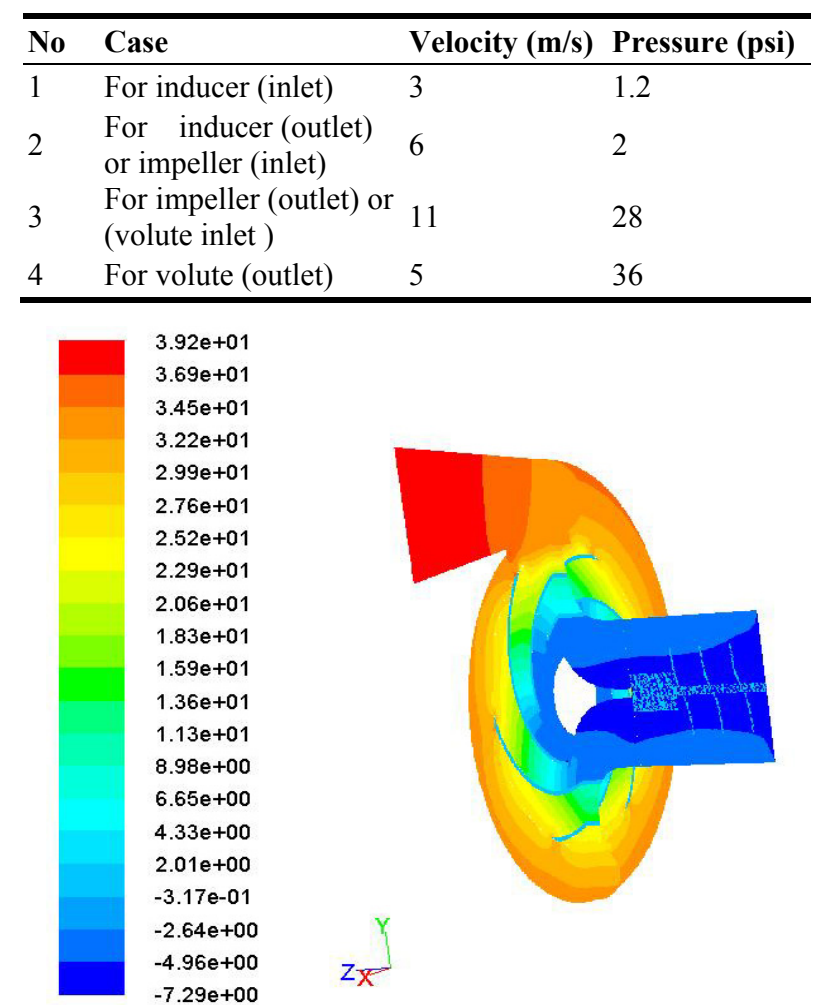

Fig. 13 Static pressure contour of case 2.

Case 3 (pump in conjunction with helical inducer): The hydraulic measurement results using helical inducer can be illustrated as follows in Table 7 and Figs. 15 and 16.

CFD model final results show that using helical inducer improved the hydraulic parameters, Figs. 15 and 16. Thus, Helical inducers improve the centrifugal

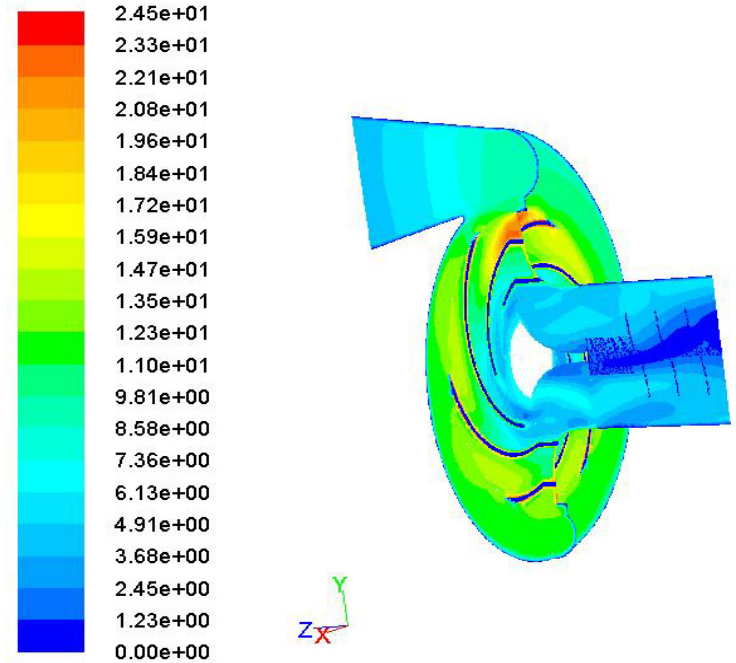

Fig. 14 Velocity contour of case 2.

Table 7 Hydraulic measurement results using helical inducer.

\begin{tabular}{llll}
\hline No. & Case & $\begin{array}{l}\text { Velocity } \\
(\mathbf{m} / \mathbf{s})\end{array}$ & $\begin{array}{l}\text { Pressure } \\
(\mathbf{p s i})\end{array}$ \\
\hline 1 & For inducer (inlet) & 3 & 1.2 \\
2 & $\begin{array}{l}\text { For inducer (outlet) or } \\
\text { impeller (inlet) }\end{array}$ & 5 & 3 \\
3 & $\begin{array}{l}\text { For impeller (outlet) or } \\
\text { (volute inlet ) }\end{array}$ & 12 & 30 \\
4 & For volute (outlet) & 4 & 39 \\
\hline
\end{tabular}
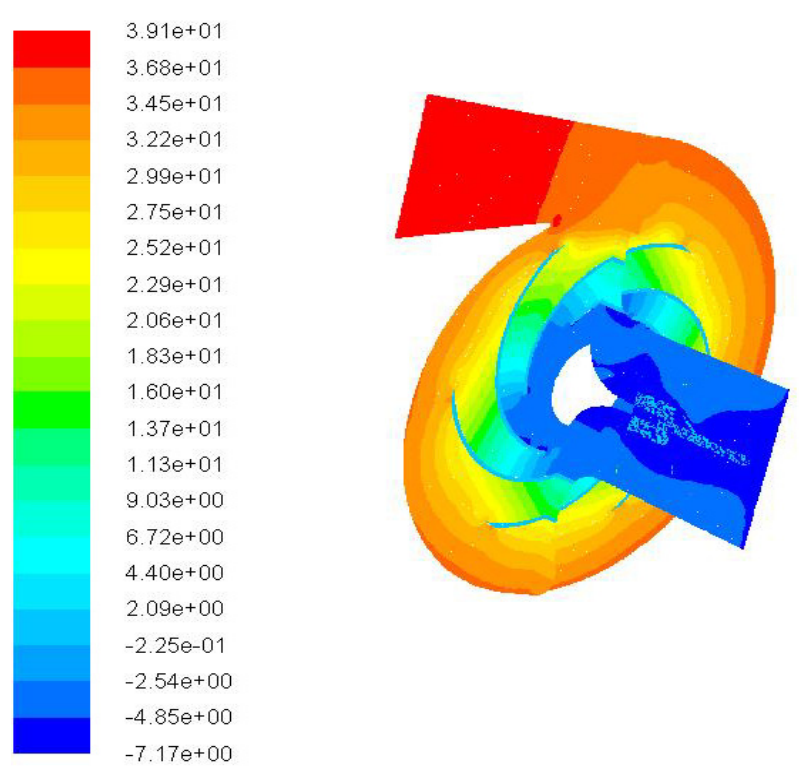

Fig. 15 Static pressure contour of case 3.

pump performance by increasing both its efficiency and head, but decreasing brake power. Therefore, this research proved that adding helical inducer enhanced the increasing of pump efficiency about $26 \%$, 

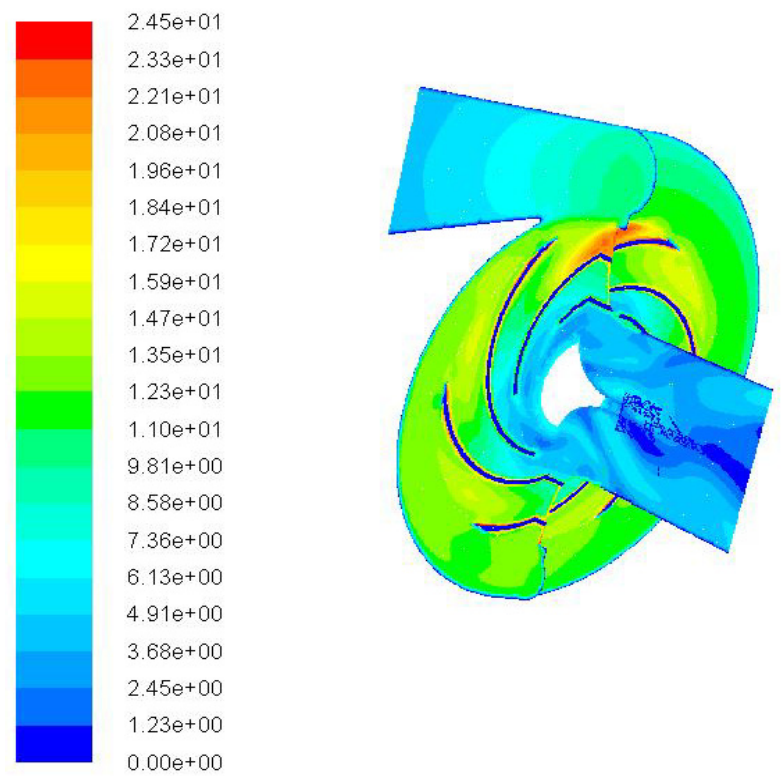

Fig. 16 Velocity contour of case 3.

increased head about 20\%, whereas using axial inducer improved the increasing of pump efficiency reach $22 \%$ and increased head about $18 \%$. A CFD model thus developed showed a good correlation with the experimentally observed values.

\subsection{NPSH (Net Positive Suction Head) for Centrifugal Pump}

One of the most important considerations in selecting and applying a centrifugal pump in the existing conditions is the pump's suction system. These conditions are best expressed as "NPSH" (net positive suction head). This term is officially defined in accordance with the standards of the Hydraulic Institute as "The total suction head in feet of liquid absolute, determined at the suction nozzle and corrected to datum, less the vapor pressure of the liquid in feet absolute". In simple terms, NPSH is the absolute pressure in feet of liquid at pumping temperature available at the pump suction flange above vapor pressure [6]. Since centrifugal pumps are incapable of handling large quantities of vapor the pump's external suction system must provide sufficient absolute pressure to prevent vaporization or flashing in the impeller. This pressure is normally referred to the centerline of the pump suction nozzle. When this pressure is not sufficient to prevent vaporization, the phenomenon known as (cavitations), occurs causing damage to the impeller, reduction in pump developed head and capacity, noise and vibration [6]. Pump manufacturers are determined to test the NPSH required at various capacities for a particular pump and plot it as a function of capacity. This is referred to as NPSHR (net positive suction head required). Conversely, the NPSH available in the system is referred to as NPSHA (net positive suction head available). For cavitations free operation the NPSHA must equal or exceed the NPSHR at the desired capacity. It must be realized that suction conditions and NPSHA are very important than the pumps capacity and total head. More attention to suction conditions and NPSHA will result in more trouble, free pump operation [6].

\subsection{Comparison between NPSH for Centrifugal Pump} with Helical, Axial, and without Inducers

In order to compare the present work with other investigators regarding the pump NPSH, the best obtained configuration of the inducers section was selected and the NPSH was calculated at these selected types at the maximum efficiency points:

(1) For helical inducer, maximum efficiency $(72 \%)$ at $1,000 \mathrm{rpm}$ is obtained by using ( 3 turns- $17^{\circ}$ ) helical inducer.

(2) For axial inducer, maximum efficiency $(62 \%)$ at $1,000 \mathrm{rpm}$ is obtained by using (4) Bladed- $\left(\beta 1=11.5^{\circ}\right.$, $\beta 2=29.5^{\circ}$ ) axial inducer.

(3) For the case without inducer, maximum efficiency (54.21\%) at 1,000 rpm.

$$
\begin{gathered}
\mathrm{NPSH}=\left\{\left(\left[(P)_{\text {air }}-(P)_{\mathrm{vapor}}\right] / \gamma\right)-H_{\mathrm{ss}}-H_{\text {loss suction }}\right. \\
\left.-\left[v^{2} / 2 \mathrm{~g}\right]\right\} 7
\end{gathered}
$$

Where,

$\mathrm{P}_{\text {air }}=1$ bar;

$\mathrm{P}_{\text {vapor }}=2.339 \mathrm{kpa}=0.02339$ bar at temp. $=30^{\circ}$;

$H_{\mathrm{ss}}$ : Vertical distance between pump and section level $=10 \mathrm{~cm}=0.1 \mathrm{~m}$; 


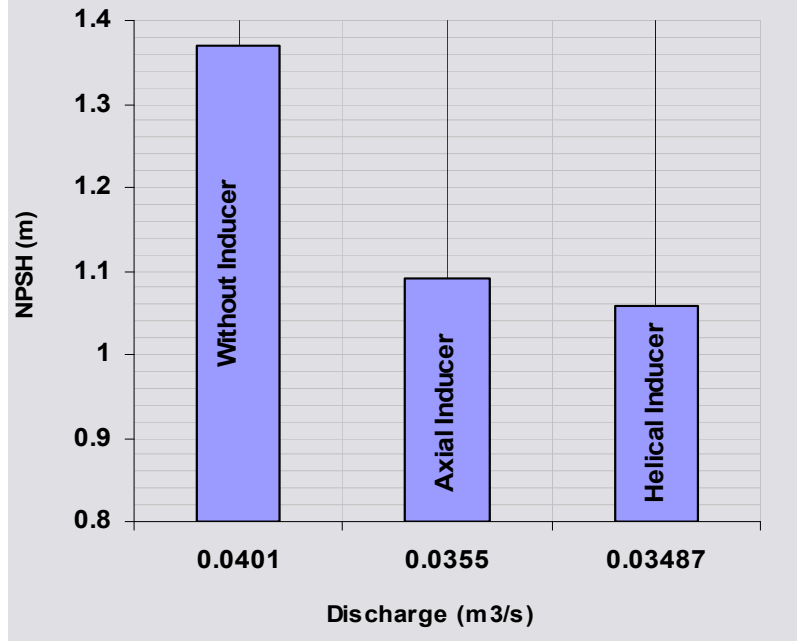

Fig. 17 Comparison between NPSH \& $Q$ for centrifugal pump with helical, axial and without inducer at $1,000 \mathrm{rpm}$.

$H_{\text {loss suction }}=\mathrm{F} \mathrm{Lv} 2 / 2 \mathrm{gd}$;

$F=$ Friction factor $=0.03$;

$L=$ Length, suction pipe $=0.8 \mathrm{~m}(80 \mathrm{~cm})$;

$d=$ Suction pipe diameter $=4 "=0.1016 \mathrm{~m}$;

$v=Q /(\Pi / 4) d^{2}$ at $(\eta \max )$.

5.3.1 Calculation of the Three Cases (Fig. 17)

\section{Case 1.}

Without inducer $\left(\eta_{\max }=54.21 \%\right.$ at $\left.1,000 \mathrm{rpm}\right)$, from equation no. (7), so; for $Q=0.0401 \mathrm{~m}^{3} / \mathrm{sec}$ and $v$ $=5.005 \mathrm{~m} / \mathrm{sec}$,

Then, $(\mathrm{NPSH})_{\text {without inducer }}=1.36931 \mathrm{~m}$.

Case 2.

For axial inducer $\left(\eta_{\max }=68 \%\right.$ at $\left.1,000 \mathrm{rpm}\right)$, from equation no. (7), So; for $Q=0.0355 \mathrm{~m}^{3} / \mathrm{sec}$ and $v=$ $4.43036 \mathrm{~m} / \mathrm{sec}$,

Then, $(\mathrm{NPSH})_{\text {Axial inducer }}=1.092318 \mathrm{~m}$.

\section{Case 3.}

For helical inducer $\left(\eta_{\max }=71.59 \%\right.$ at $\left.1,000 \mathrm{rpm}\right)$, from equation no. (7), so; for $Q=0.03487 \mathrm{~m}^{3} / \mathrm{sec}$ and $v=4.35174 \mathrm{~m} / \mathrm{sec}$,

Then, $(\mathrm{NPSH})_{\text {Helical inducer }}=1.057044 \mathrm{~m}$.

\subsubsection{Final Results; from Fig. 17}

It's so clear that adding an inducer can improve pump performance against cavitation. Moreover, the helical inducer exhibits the best performance against cavitation; the second is the axial inducer; the third is the pump without inducer.

\section{Conclusions}

The flow of the centrifugal pump with inducers: axial (4-blades) and helical (3 turns) are numerically simulated. The corresponding performance is carried out experimentally. The conclusions can be summarized as follows:

Comparison of the simulation and experiment shows that the trends of performance curves are close. The tested pump conjunction with different inducers has different heads. The head of the centrifugal pump reaches its highest values with using helical inducers;

Moreover, using helical inducer enhances pump hydraulic performance as head increased $20 \%$. Using axial inducer enhances pump hydraulic performance as head increased $18 \%$. On the other hand, the pump should be mechanically compatible with the addition of inducer otherwise it may produce a problem. Using helical inducer enhances both dynamic and hydraulic performances of the pump with an insignificant change than using axial inducer, but with high obviously values than using pump without inducer;

The present research paper can supply significant guide for inducer's user for different applications.

\section{References}

[1] Anton, L. 2008. "Numerical and Experimental Investigations of the Flow into an Inducer." In Proceedings of the International Conference on Hydraulic Machinery and Equipment, 1-6.

[2] Wilox, E. 2007. "Reliability Improvements to a High Speed/High Energy Pump." In Proceedings of the Twenty-Third International Pump Users Symposium, 1-6.

[3] Lee, S., Jung, K.-H., Kim, J.-H., and Kang, S.-H. 2002. "Cavitation Mode Analysis of Pump Inducer." KSME International Journal 16: 1497-510.

[4] Ashihara, K., and Goto, A. 2002. "Effects of Blade Loading on Pump Induce Performance and Flow Fields." In Proceedings of ASME Fluid Engineering Division Summer Meeting, 925-34.

[5] Mostafa, M. A. A. 2012 "Experimental \& Theoretical Investigations on the Performance of Centrifugal Pump in Conjunction with Inducers." Ph.D. thesis, AinShams University. 
Reliability Improvements to Centrifugal Pump Performance in Conjunction with

\section{Inducers, CFD Comparative Study}

[6] Moawad, M. A. 2015. "Study of Improving a Centrifugal Performance." Master thesis, Benha University.

[7] Munson, B. R. 2001. Fundamental of Fluid Mechanics. New York: John Wiley and Sons.
[8] Cheah, K. W., Lee, T. S., Winoto, S. H., and Zhao, Z. M. 2007. "Numerical Flow Simulation in a Centrifugal Pump at Design and Off-Design Conditions." International Journal of Rotating Machinery 1: 1-8. 